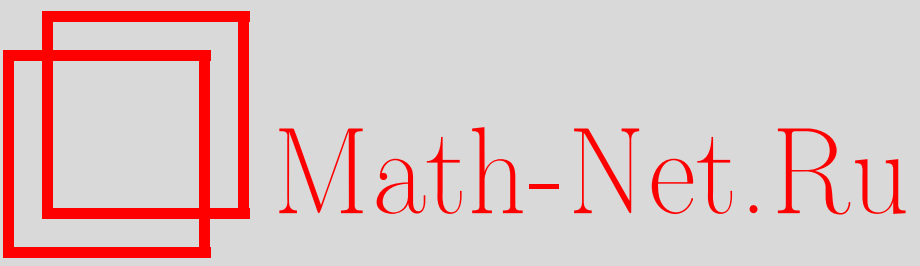

М. А. Лифшиц, Е. Ю. Шмилева, Критерий квазиинвариантности пуассоновских мер относительно "линейных" трансформаций пространства, УМН, 2001, том 56, выпуск 6, 159-160

DOI: https://doi.org/10.4213/rm465

Использование Общероссийского математического портала Math-Net.Ru подразумевает, что вы прочитали и согласны с пользовательским соглашением

http://www. mathnet.ru/rus/agreement

Параметры загрузки:

IP: 54.237 .206 .68

26 апреля 2023 г., 13:07:32 


\title{
КРИТЕРИЙ КВАЗИИНВАРИАНТНОСТИ \\ ПУАССОНОВСКИХ МЕР ОТНОСИТЕЛЬНО “ЛИНЕЙНЫХ” ТРАНСФОРМАЦИЙ ПРОСТРАНСТВА
}

\author{
М. А. ЛИФШиц, Е. Ю. ШмилЕВА
}

Пусть $(X, \mathscr{F}, \nu)$ - произволное измеримое пространство, где $\nu$ - конечная неатомическая мера. Рассмотрим продакт-пространство $X \times \mathbb{R}^{+}$с $\sigma$-алгеброй $\mathscr{F} \times \mathscr{B}$ и мерой $\nu \times \Lambda$, где $\mathscr{B}-$ борелевская $\sigma$-алгебра на $\mathbb{R}^{+}$, а $\Lambda$ - произвольная мера на $\mathbb{R}^{+}$. Исследуемая задача состоит в том, чтобы найти условия, описьвающие распределения всех пуассоновских мер со спектральными мерами вида $\nu \times \Lambda$, квазиинвариантные относительно достаточно широкой группы растяжений пространства $X \times \mathbb{R}^{+}$по одномерной компоненте. Уточним, какой класс преобразований нас интересует. Для этого введем обозначение для пространства целочисленных мер на $X \times \mathbb{R}^{+}$ $\mathscr{M}=\left\{\eta: \forall A \in \mathscr{F} \times \mathscr{B} \quad \eta(A) \in \mathbb{Z}^{+}\right\}$, а также для множества неотрицательных измеримых функций на пространстве $X$ с $\nu$-суммируемьп логарифмом

$$
\Phi=\left\{a: X \rightarrow \mathbb{R}^{+}: \int_{X}|\ln a(x)| d \nu(x)<\infty\right\} .
$$

Далее, каждой функции $a \in \Phi$ сопоставим преобразование $\mathscr{L}^{a}: X \times \mathbb{R}^{+} \rightarrow X \times \mathbb{R}^{+}$, которое действует на $(x, r) \in X \times \mathbb{R}^{+}$так: $\mathscr{L}^{a}(x, r)=(x, a(x) r)$. При этом в пространстве мер определим преобразование $\mathscr{L}_{a}: \mathscr{M} \rightarrow \mathscr{M}$, которое каждой мере $\phi \in \mathscr{M}$ сопоставляет $\mathscr{L}_{a} \phi(B)=\phi\left(\left(\mathscr{L}^{a}\right)^{-1}(B)\right)$, где $B \in \mathscr{F} \times \mathscr{B}$. Множество $\left\{\mathscr{L}_{a}\right\}_{a \in \Phi}$ является группой "линейных" трансформаций пространства $\mathscr{M}$. Квази-инвариантность распределений пуассоновских мер относительно этой группы преобразований и будет нас интересовать в дальнейшем. Об этой задаче впервые упоминалось в работах А. М. Вершика, И. М. Гельфанда и М. И. Граева [1] и [2] в связи с представлениями группы ограниченных измеримых функций на многообразии с гладкой мерой со значениями в $S L(2, \mathbb{R})$, позднее - в ряде работ А. М. Вершика, Н. В. Цилевич и М. Йоpa [3], [4], в которых исследуются свойства гамма-процесса и мер Пуассона-Дирихле в контексте теории представлений. Следующая теорема содержит искомьй критерий квазиинвариантности.

ТЕорема. Пуассоновская мера является квазиинвариантной относительно всех преобразований $\left\{\mathscr{L}_{a}\right\}_{a \in \Phi}$ тогда и только тогда, когда ее спектральная мера представима в виде

$$
d m(x, r)=d \nu(x) \cdot \frac{\psi^{2}(\ln r)}{r} d r
$$

где функция $\psi$ удовлетворяет следующим условиям:

1) $\psi(s)$ строго положительна при почти всех $s \in \mathbb{R}$;

2) при некотором $c>0$

$$
\int_{|v|>r}|\widehat{\psi}(v)|^{2} d v \leqslant c(1+1 / r) \quad \forall r>0
$$

где $\widehat{\psi}$ - преобразование Фурье функции $\psi$.

Проиллюстрируем эту теорему несколькими примерами. А. М. Вершик с соавторами рассматривал гамма-меру, квазиинвариантность которой была ими установлена с использованием преобразования Лапласа мер, возникающих при трансформациях пространства (см. [3], [4]).

ОПРЕДЕЛЕнИЕ. Гамма-мерой называется закон распределения в пространстве $\mathscr{M}$ случайной пуассоновской меры на пространстве $X \times \mathbb{R}^{+}$со спектральной мерой

$$
d m(x, r)=d \nu(x) \cdot \frac{e^{-r}}{r} d r .
$$

Работа первого автора поддержана грантами РФФИ № 00-15-96019 и № 99-01-00112. 
До сих пор не было известно, есть ли в классе распределений пуассоновских мер, сосредоточенных на множестве $\{\mu \in \mathscr{M}: \mu((0, \varepsilon) \times \mathscr{X})=\infty\}(\varepsilon$ - любое сколь угодно малое положительное число $)^{1}$, квазиинвариантные меры, сингулярные относителшно гамма-меры. Теорема позволяет доказать квазиинвариантность распределений пуассоновских мер со спектральными плотностями вида

$$
\phi_{\alpha}(r)= \begin{cases}\frac{1}{r|\ln r|^{\alpha}}, & r \in(0, \varepsilon), \\ g(r), & r \in[\varepsilon, \infty),\end{cases}
$$

где $\varepsilon<1, \alpha \in[0,1]$, а $g$ - любая строго положителшная суммируемая на $[\varepsilon, \infty)$ функция. В этом семействе при $\alpha=0$ получается мера, эквивалентная гамма-мере. Во всех остальных случаях меры сингулярны относительно гамма-меры, что и подтверждает неединственность гамма-меры в классе рассматриваемых мер. Условия теоремы выполнены и для класса мер, задаваемого спектральньми мерами вида $d \nu(x) \cdot \frac{\psi^{2}(\ln r)}{r} d r$, где $\psi(v) \in \Psi$ при $v \in(-\infty, \ln \varepsilon)$ и $\psi$ - любая строго положительная квадратично суммируемая на интервале $[\ln \varepsilon, \infty)$. Здесь $\varepsilon<1$ и

$$
\Psi=\left\{\varphi \in W_{1}^{1}(-\infty, \ln \varepsilon):\left|\varphi^{\prime}(v)\right| \leqslant \frac{c}{|v|} \text { и } 0<\varphi(v)<c_{0}, c_{0}, c>0\right\} .
$$

Этот класс включает в себя и гамма-меру, и все меры со спектральными плотностями вида (1). Рассмотрим функции из $\Psi$, не имеющие предела при $v \rightarrow-\infty$, например, $\psi_{1}(v)=\cos \ln (|v|+1)+$ $c$, где $c>1, v \in(-\infty, 0)$, или кусочно линейную функцию

$$
\psi_{2}(v)= \begin{cases}\frac{1}{2^{2 k}} v+3, & v \in\left[-2^{2 k+1},-2^{2 k}\right), k=0,1, \ldots, \\ -\frac{1}{2^{2 k-1}} v, & v \in\left[-2^{2 k},-2^{2 k-1}\right), k=1,2, \ldots, \\ -v+1, & v \in[-1,0] .\end{cases}
$$

Этим функциям соответствуют пуассоновские меры со спектральными плотностями, медленно меняющимися между плотностями гамма-мер разного масштаба, а значит, сингулярные относительно любой гамма-меры. Таким образом, предъявлен еще более широкий класс мер, квазиинвариантных относительно $\left\{\mathscr{L}_{a}\right\}_{a \in \Phi}$, которьй содержит множество мер, сингулярных относителшно гамма-меры. Теорема была нами доказана как частный случай критерия взаимной абсолютной непрерьвности двух пуассоновских мер на произвольном измеримом пространстве. Столь общий случай уже был изучен А. В. Скороходом (см. [5]), но лишь для процессов с независимьми приращениями. Удобные для проверки условия абсолютной непрерьвности однородных процессов с независимыми приращениями были предложены также в работе [6].

\section{СПИСОК ЛИТЕРАТУРЫ}

[1] I. M. Gelfand, M. I. Graev, A. M. Vershik. Models of representations of current groups // Representations of Lie Groups and Lie Algebras / ed. A. A. Kirillov. Budapest: Akadémiai Kiadó, 1985. Р. 121-179. [2] И. М. Гельфанд, М. И. Граев, А. М. Вершик // Функц. анализ и его прил. 1983. T. 17. № 2. С. 70-72. [3] N. Tsilevich, A. Vershik, M. Yor // C. R. Acad. Sci. Paris. Sér. I Math. 1999. V. 329. P. 163-168. [4] N. Tsilevich, A. Vershik, M. Yor. Distinguished properties of the gamma process and related topics // Prepublication № 575 du Laboratoire de Probabilités et Modèles Aléatoires. 2000. [5] А. В. Скороход. Случайные процессы c независимыми приращениями. М.: Наука, 1964. [6] C. M. Newman // Bull. Amer. Math. Soc. 1972. V. 78. P. 268-272.

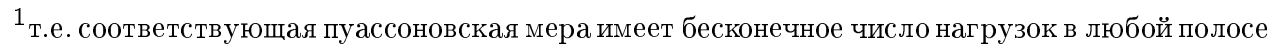
около нуля.
} 